\title{
Observation on the Effect of Digitalis on Sinus Node Function by Recording of Twenty-four Hour Continuous Electrocardiogram
}

\author{
Kuniaki Orsuka, M.D. and Takashi YanagA, M.D.
}

\section{SUmmary}

Twenty-four hour recording of electrocardiogram was performed in 5 cases, aged from 42 to 75 years, with paroxysmal tachyarrhythmias (paroxysmal supraventricular tachycardia (PSVT) 2 cases and paroxysmal atrial fibrillation (PAf) 3 cases) for the purpose of assessing the effects of digitalis on sinus node function from the chronobiological point of view. All of the pauses following the termination of PSVT or PAf observed during a day were measured in 4 cases as the sinus node recovery time (SNRT). Corrected sinus node recovery time (CSNRT) was determined by subtracting the penultimate P-P cycle length from the SNRT. Both SNRT and CSNRT were prolonged in all cases after digitalis administration. In 1 case observation on the diurnal variation of SNRT and CSNRT showed the most marked prolongation of these values at 6:00 a.m. after digitalis administration. The second degree of SA block was observed in 2 cases in the early morning after digitalis treatment. In the other 2 cases sinoatrial conduction time (SACT) was calculated by the use of premature atrial contraction and corrected by dividing by the basic cycle length before and after digitalis administration. Neither SACT and CSACT showed significant differences between the values before and after the treatment in 2 cases, nor the diurnal variation of SACT and CSACT showed significant differences. Above results suggest that digitalis should be used with caution in patients with paroxysmal tachyarrhythmia because even small amounts of digitalis might provoke sinus node dysfunction. Twenty-four hour recording of electrocardiogram is useful in the earlier recognition of sinus node dysfunction induced by digitalis.

\section{Additional Indexing Words:}

Paroxysmal supraventricular tachycardia Paroxysmal atrial fibrillation Sinus node recovery time Corrected sinus node recovery time Sinoatrial conduction time Corrected sinoatrial conduction time SA block Chronobiology

GEVERAL recent reports ${ }^{1,2)}$ have suggested that responses to brief periods $\infty$ of rapid atrial stimulation or to single premature atrial stimulus can be useful in evaluating the function of the sinoatrial node. These techniques

From the Department of Bioclimatology and Medicine (Director Prof. Masaro Kaji), Institute of Balneotherapeutics, Kyushu University, Tsurumihara 4546, Beppu, Oita 874, Japan.

Received for publication August 6, 1976. 
have been applied for the evaluation of patients with the clinical diagnosis of sinus node disease and for the assessment of drug effects in these patients.

Margolis $\mathrm{et}^{\mathrm{a}} \mathrm{l}^{31}$ have also reported that overdrive pacing elicited the definite abnormality of sinus node function following the administration of a moderate amount of digoxin.

In the previous paper, we ${ }^{4}$ have reported the usefulness of 24-hour monitoring of electrocardiogram in the observations of cardiac rhythm. However the diurnal variation of sinus node function under the digitalis action remains to be clarified.

The purpose of this study was to assess the effects of digitalis on sinus node function especially from the chronobiological point of view by 24-hour recording of electrocardiogram.

\section{Methods}

Five patients ranging in age from 42 to 75 years, with paroxysmal supraventricular tachycardia (PSVT) or paroxysmal atrial fibrillation (PAf) were evaluated for sinus node dysfunction before and after digitalis administration. The patients were given digitoxin 0.05 or $0.1 \mathrm{mg} /$ day.

Twenty-four hour electrocardiogram was recorded with Holter Dynamic Electrocardiographic System (Avionics Research Products, Los Angeles, California). A bipolar electrode lead system was used. The remote lead was located over the right upper area of the manubrium and the exploring lead was located in the left anterior axillary line over the fifth intercostal space. A ground lead was placed in the right midclavicular line at the costal margin.

All of the pauses following the termination of PAf or PSVT observed during a day were measured as the sinus node recovery time (SNRT); they were defined as the interval, in sec, from the last $\mathrm{P}$ wave of PSVT or from the last $\mathrm{R}$ wave of PAf to the first spontaneously occurring $\mathrm{P}$ wave. Corrected sinus node recovery time (CSNRT) was determined by subtracting the penultimate P-P cycle length from the SNRT. SNRT and CSNRT were measured in 4 cases before and after digitalis administration.

Table I. Clinical Data of Patients

\begin{tabular}{|c|c|c|c|c|c|}
\hline Case No. & Name & Age (yrs) & Sex & Cardiac Drugs & Cardiac Diagnosis \\
\hline 1 & M. K. & 63 & M & $0.05 \mathrm{mg}$ & PSVT, VSD \\
\hline 22 & H. T. & 75 & M & $0.075 \mathrm{mg}$ & PAf, IHD \\
\hline 3 & I. Y. & 70 & M & $0.075 \mathrm{mg}$ & PSVT, IHD \\
\hline 4 & E. $H$. & 42 & $\mathbf{M}$ & $0 \cdot 1$ & PAf, IHD \\
\hline 5 & T. N. & 60 & $\mathrm{M}$ & $0.05 \mathrm{mg}$ & PAf, IHD \\
\hline
\end{tabular}

Abbreviations: $\quad M=$ male, PSVT = paroxysmal supraventricular tachycardia, $P A f=$ paroxysmal atrial fibrillation, $\mathrm{VSD}=$ ventricular septal defect, $\mathrm{IHD}=$ ischemic heart disease. 
Sinoatrial conduction time (SACT) was calculated by the use of all premature atrial contractions observed by 24-hour electrocardiogram. As described by Strauss et al, ${ }^{51,6)}$ reset of the sinus node by an atrial premature contraction occurred when the sum of the atrial premature cycle and the return cycle was less than the sum of 2 spontaneous sinus cycles, and in this zone SACT can be estimated as onehalf of the difference between atrial return cycle and basic cycle length. SACT was corrected by dividing by the basic cycle length (CSACT). SACT and CSACT were calculated in 2 cases before and after digitalis administration.

\section{RESULTS}

In 4 cases (Cases 1, 2, 3, and 4) SNRT and CSNRT were measured before and after digitalis aministration, as shown in Fig. 1. SNRT and CSNRT were prolonged in all cases after digitalis administration, that is, SNRT ranged from 0.64 to $1.92 \mathrm{sec}$ before and ranged from 0.80 to $4.28 \mathrm{sec}$ after digitalis treatment. CSNRT ranged from -0.18 to $1.00 \mathrm{sec}$ before and ranged from -0.26 to $3.08 \mathrm{sec}$ after the drug administration. The negative value for CSNRT was due to a sinus escape beat that appeared earlier than
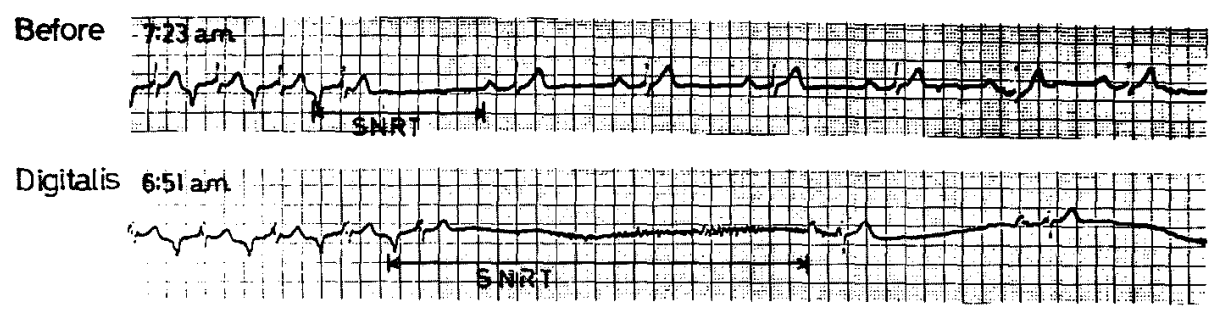

\begin{tabular}{|c|c|c|c|c|c|c|c|c|c|c|c|}
\hline \multirow[b]{2}{*}{ Name } & \multirow[b]{2}{*}{ Age } & \multirow[b]{2}{*}{ Sex } & \multirow[b]{2}{*}{ Digitalis } & \multicolumn{4}{|c|}{ SNRT sec } & \multicolumn{4}{|c|}{ corrected SNRT sec } \\
\hline & & & & $n$ & Mean & S.D. & Range & $n$ & Mean & S.D. & Range \\
\hline \multirow{2}{*}{ M.K. } & \multirow{2}{*}{63} & \multirow{2}{*}{$M$} & before & 12 & 1.53 & 0.22 & $1.16-1.92$ & 12 & 0.57 & 0.23 & $0.20-1.00$ \\
\hline & & & after & 58 & 200 & 0.42 & $1.62-4.28$ & 55 & 1.00 & 0.40 & $0.66-308$ \\
\hline \multirow{2}{*}{ H. T. } & \multirow{2}{*}{75} & \multirow{2}{*}{1} & before & 2 & 1.12 & 0,06 & $108-1.16$ & 2 & 0.12 & 0.06 & $008-016$ \\
\hline & & & after & 16 & 1.39 & 0.29 & $080-1.78$ & 15 & 0.28 & 0.30 & $-0.26-0.60$ \\
\hline \multirow{2}{*}{ I. Y. } & \multirow{2}{*}{70} & \multirow{2}{*}{$M$} & before & 3 & 0.83 & 0.18 & $0.64-1.00$ & 2 & $-0,07$ & 0.16 & $-0.18-0.06$ \\
\hline & & & after & 3 & 1.80 & 0.85 & $0.96-266$ & 3 & 0.86 & 0.97 & $-0.16-1.78$ \\
\hline \multirow{2}{*}{ E.H. } & & & before & 10 & 0.91 & 0.16 & $0.76-1.28$ & 8 & 0.03 & 0.14 & $-0.12-0.22$ \\
\hline & & & after & 3 & 1.38 & 0.30 & $1.06-1.50$ & 3 & 0.53 & 0.28 & $0.20-0.70$ \\
\hline
\end{tabular}

Fig. 1. The upper panel shows electrocardiogram recorded during the termination of supraventricular tachycardia before and after digitoxin. After the administration of digitoxin the cessation of the tachycardia is followed by a marked suppression of sinus node function. The lower panel shows the values of SNRT and CSNRT before and after digitoxin in patient MK (Case 1), HT (Case 2), IY (Case 3), and EH (Case 4). CSNRT was determined by subtracting the penultimate P-P cycle length from the SNRT. After digitoxin administration SNRT and CSNRT were prolonged in all cases. 


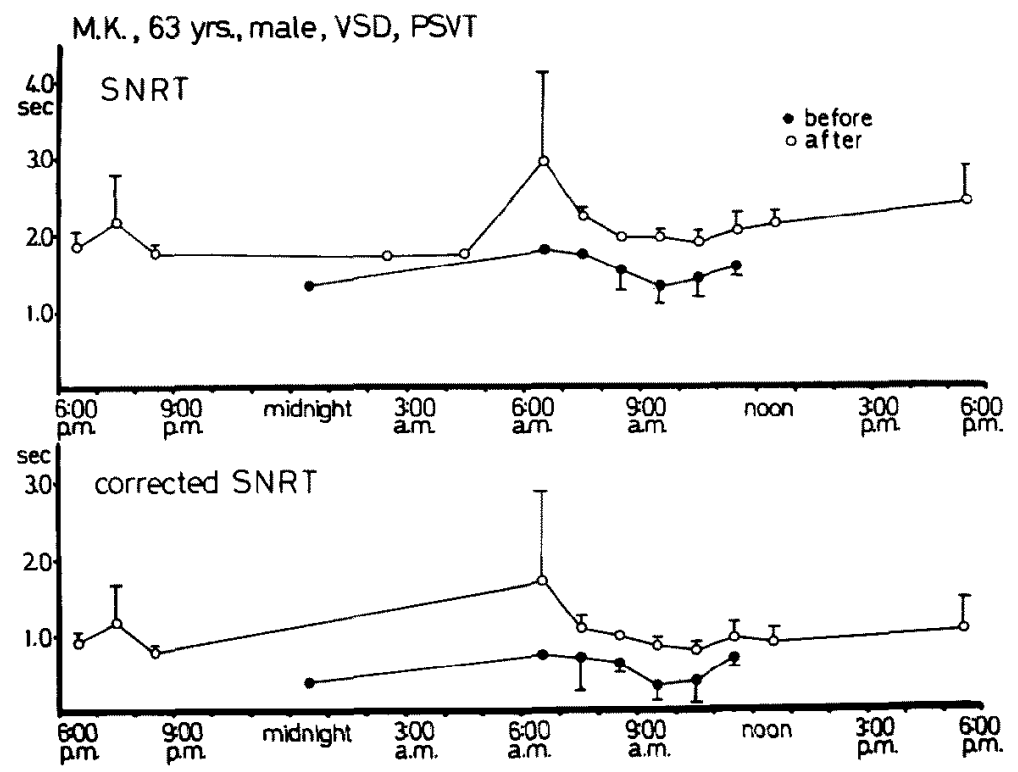

Fig. 2. Diurnal variation of SNRT and CSNRT in patient MK (Case 1). Note that both SNRT and CSNRT were prolonged during a day after digitoxin administration, and that the most marked prolongation of the SNRT and CSNRT was observed at 6:00 a.m.

the average sinus cycle length (that is, SNRT was less than the penultimate $\mathrm{P}-\mathrm{P}$ interval). The variation in SNRT and CSNRT was observed during a day in the same case. The diurnal variation of SNRT and CSNRT in Case 1 was shown in Fig. 2. Although in this case the prolongation of SNRT as well as CSNRT was observed during a day after digitalis administration, it was marked in the early morning.

After digitalis treatment the second degree of sinoatrial block was observed in Case 1 and Case 4 only in the early morning by 24-hour electrocardiogram.

Moreover because premature atrial contractions were frequently recorded by 24-hour electrocardiography in Case 2 and Case 5, SACT was calculated by the use of premature atrial contraction before and after digitalis administration, as shown in Fig. 3. SACT ranged 90 to $180 \mathrm{msec}$ before and ranged from 90 to $210 \mathrm{msec}$ after digitalis treatment. CSACT ranged from 11.3 to $19.8 \%$ before and ranged from 11.0 to $23.7 \%$ after the drug administration. The diurnal variation of SACT and CSACT was shown in Fig. 4 in Case 2, but no significant difference between the values before and after the treatment was found. 


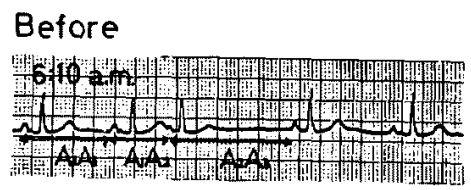

H.T. 75yrs. male

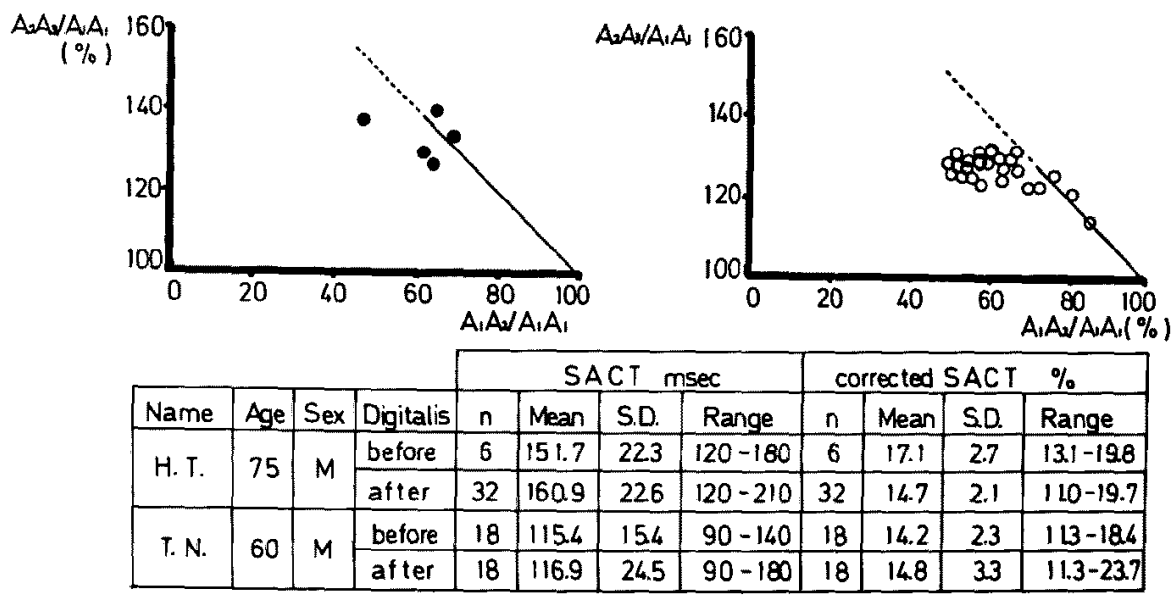

Fig. 3. The upper panel shows atrial premature beats before and after digitoxin administration. In the middle panel the return cycles are plotted as a function of the premature cycles for patient HT (Case 2) before and after digitoxin. The lower panel shows the values of SACT and CSACT before and after digitoxin administration in patient $\mathrm{HT}$ (Case 2) and TN (Case 5). CSACT was determined by dividing by the basic cycle length. Not significant differences were shown between the values of before and after digitoxin administration.

\section{Discussion}

Margolis et $\mathrm{al}^{31}$ have reported that digitalis, in a common clinical dose and at a low serum level, brought out severe manifestations of sinus node dysfunction in a patient who had previously undergone successful mitral valve replacement and that digitalis preparations should be used with caution because even small amounts of this agent may be extremely toxic for selected patients.

In our cases a definite prolongation of the initial spontaneous sinus cycle following paroxysmal tachyarrhythmias was observed after the administration of digitoxin $0.05-0.1 \mathrm{mg}$ per day with 24-hour continuous electrocardiography.

On the other hand, recently the evaluation of sinus node function in the in situ human heart has been attempted by 2 methods. The pacemaker function of the sinus node has been evaluated by observing the degree of postpacing depression after rapid atrial stimulation. Conduction from the sinus 


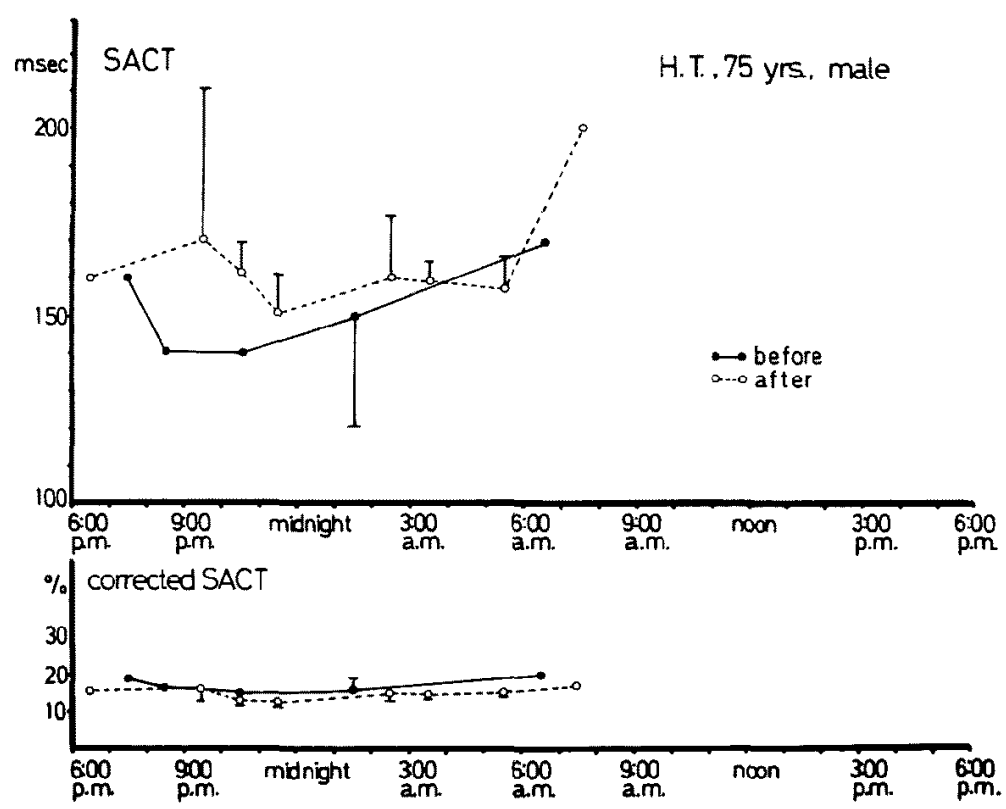

Fig. 4. Diurnal variation of SACT and CSACT in patient HT (Case 2). There was no significant difference before and after digitoxin administration during a day.

node to the atrium has been estimated by the use of premature atrial stimulation. However these techniques have the following problems: (1) To evaluate the sinus node function by analyzing the sinus node recovery time there must be discussed about the effects of pacing rate, pacing duration, milliamperage, vagal and beta-adrenergic stimulation and sinus node injury. (2) The sinoatrial conduction time is calculated assuming that the sinoatrial node pacemaker cycle is equal to the spontaneous sinus cycle. ${ }^{7}$ (3) The conduction time from the sinoatrial node to the atrium is equal.

In this paper the sinus node recovery time observed in terminating repetitive supraventricular tachycardia or atrial fibrillation by 24-hour continuous electrocardiography was used to evaluate its automatic pacemaker function instead of rapid atrial pacing or programmed atrial stimulation. The calculation of the sinoatrial conduction time was made by the use of spontaneous atrial premature contractions observed by 24-hour electrocardiography. This technique has an advantage in that the sinus node function is estimated in the natural and clinical state, acetylcholine release or an increase in parasympathetic tone by atrial pacing need not be discussed and only endogenous factors might be important in evaluating sinus node function.

Moreover this technique allows an evaluation of diurnal variation in the degree of sinus node dysfunction. The most marked prolongation of the 
SNRT was observed at 6:00 a.m. in a patient who was observed marked prolongation of SNRT after administration of digitoxin $0.05 \mathrm{mg}$ per day. In 2 cases second degree of sinoatrial block was also observed at night. The mechanism of this diurnal variation is not clear but probably mediated in increase of parasympathetic tone at night. Already we ${ }^{8)}$ have reported that major arrhythmias were frequently observed at night or in the early morning during digitalis administration. These results might suggest that the sensitivity to digitalis was increased in the early morning.

In conclusion digitalis should be used with caution in patients with paroxysmal tachyarrhythmias because even small amounts of digitalis might provoke sinus node dysfunction. Twenty-four hour recording of electrocardiogram is useful in the observation on the diurnal variation of the effects of digitalis on sinus node function.

\section{RefERENCES}

1. Mandel W, Hayakawa H, Danzig R, Marcus HS: Evaluation of sino-atrial node function in man by overdrive suppression. Circulation 44:59, 1971

2. Narula OS, Samet $\mathbf{P}$, Javier RP: Significance of the sinus node recovery time. Circulation 45: 140,1972

3. Margolis JR, Strauss HC, Miller HC, Gilbert M, Wallace AG: Digitalis and the sick sinus syndrome. Clinical and electrophysiologic documentation of a severe toxic effect on sinus node function. Girculation 52: 162, 1975

4. Yanaga $\mathrm{T}$, Otsuka $\mathrm{K}$, Tsubone $\mathrm{T}$, Yoshioka $\mathrm{M}$ : Diurnal variation of cardiac rhythm studied by recording of longterm continuous electrocardiogram. Jap Circulat J 38: 595, 1974

5. Strauss HC, Saroff AL, Bigger JT Jr, Giardina EGV: Premature atrial stimulation as a key to the understanding of sino-atrial conduction in man. Circulation 47:86, 1973

6. Miller HC, Strauss HC: Measurement of sinoatrial conduction time by premature atrial stimulation in the rabbit. Circulat Res 35: 935, 1974

7. Breithardt G, Seipel L: The effect of premature atrial depolarization on sinus node automaticity in man. Circulation 53: 920, 1976

8. Otsuka K, Murase K, Itoyama Y, Yanaga $\mathrm{T}$ : Two cases with arrhythmias observed at night by long-term continuous electrocardiography during digitalis treatment. Int Med 37: 475,1976 